\title{
A Comparison of Methods for Examining the Effect of Uncertainty in the Conductivities in a Model of Partial Thickness Ischaemia
}

\author{
Barbara M Johnston $^{1}$, Akil Narayan ${ }^{2}$, Peter R Johnston ${ }^{1}$ \\ ${ }^{1}$ Griffith University, Nathan, Queensland, Australia \\ ${ }^{2}$ University of Utah, Salt Lake City, Utah, USA
}

\begin{abstract}
Although models of cardiac ischaemia require values for electrical conductivities, no agreement yet exists on these values, nor how much effect uncertainty in the conductivities will have on simulation results. In this work, simulations of acute partial thickness $(50 \%$ of the wall thickness) ischaemia are conducted in a half-ellipsoidal model of the left ventricle to produce epicardial potential distributions (EPDs), while simultaneously varying each of the six bidomain conductivity values. From these, emulators are produced using Gaussian Process emulators (GPE), Partial Least Squares (PLS), and generalised polynomial chaos expansion (PCE). All three approaches indicate that the EPDs are most sensitive to uncertainty in the longitudinal conductivities and the extracellular normal conductivity, and that variation in these conductivities has a significant effect on the EPDs. Varying the depth of the ischaemic region does not change the conductivities to which the main EPD features (a maximum and two flanking minima) are sensitive. For this work, the PCE approach has the advantage that it provides more information (that is, sensitivity over the ventricular surface, not just at the main features). However, GPE and PLS are able to supply information about the sensitivity of the position of the features that PCE is not. Finally, PLS and PCE can be performed using fewer points than for GPE.
\end{abstract}

\section{Introduction}

A number of modelling studies [1,2] have suggested that uncertainty, in the electrical conductivity values that are inputs to bidomain models of cardiac ischaemia, may have a significant effect on the potentials that are produced on the epicardium, whereas other work $[3,4]$ has found that the depth of ischaemia through the ventricular wall and the rotation angle of the cardiac fibres have a more significant effect than the conductivity values. This study has examined the importance of the conductivity values in the context of personalised medicine - that is, for a certain fixed

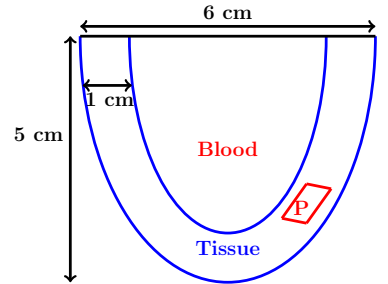

Figure 1. Cross-section $(x-z$ plane at $y=0, x \rightarrow$ and $z \downarrow$ ) of the half-ellipsoidal model, with partial ischaemic region marked with the letter ' $\mathrm{P}$ '.

ischaemic depth, could differences in conductivity values across the population result in significantly different EPDs (and therefore possibly ECGs)?

Here this is investigated via the use of emulators (GPE, PLS and PCE), which provide fast-running surrogate models as an alternative to conducting large numbers of simulations. In this work, we model acute partial thickness ischaemia, where ischaemia does not begin at the endocardium, but rather partway through the ventricular wall, in accord with a recent experimental study by Aras et al. [5]. The simulations are carried out during the ST-segment of the ECG using the bidomain model, where the conductivities that are varied are denoted by $g_{p q}$, where $p=i, e$, for the intra- and extracellular domains, and $q=l, t, n$ for the direction of conduction, longitudinal $(l)$, transverse $(t)$, normal $(n)$, where the $t$ and $n$ directions are orthogonal to the $l$ direction along the cardiac fibres, within and between the sheets of fibres, respectively. In addition to quantifying the effect of the conductivity values on the EPDs, this study also compares the information produced by the three approaches, as well as their advantages and disadvantages.

\section{Methods}

This study models the electric potential in a halfellipsoidal model of the left ventricle, which is filled with blood (Figure 1), during the iso-electric ST-segment of the ECG, via the passive bidomain equation [6] and Laplace's 
equation in the blood,

$$
\nabla \cdot\left(\mathbf{M}_{i}+\mathbf{M}_{e}\right) \nabla \phi_{e}=-\nabla \cdot \mathbf{M}_{i} \nabla \phi_{m} \text { and } \nabla^{2} \phi_{b}=0 \text {. }
$$

Here $\phi_{e}, \phi_{m}$ and $\phi_{b}$ are the extracellular, transmembrane and blood potentials, respectively, and $\mathbf{M}_{p}(p=i, e)$ are tensors that incorporate both the bidomain conductivity values and the linear rotation of the cardiac fibres through the ventricular wall via the fibre rotation angle ROT [3]. For these simulations, unless otherwise mentioned, the ischaemic region comprises $50 \%$ of the ventricular wall, from $20 \%$ to $70 \%$ depth from the endocardium, and is of size $20^{\circ}\left(x-z\right.$ plane) by $30^{\circ}(y-z$ plane) (see $[3,4]$ for more details). Mean parameter values that come from the literature [7] are used in the simulations as follows: $\left(g_{i l}=\right.$ $g_{\text {el }}=2.4, g_{i t}=0.24, g_{\text {et }}=1.6, g_{\text {in }}=1.0, g_{\text {en }}=0.1$, blood conductivity $\left.g_{b}=6.5\right) \mathrm{mS} / \mathrm{cm}, \mathrm{ROT}=100^{\circ}$. See [3] for further details such as how acute ischaemia is modelled, a 'narrow' transmembrane potential distribution is used, and for details of the solution technique.

For the GPE and PLS methods, the model is solved for $N$ sets of inputs, where the six bidomain conductivities vary between sets, while ROT, $g_{b}$ and the ischaemic depth are fixed as detailed above. Using these, $N$ EPDs are produced, from which values for the potentials of the minima and maxima are extracted and emulators constructed, using the software GP_emu_UQSA (DOI 10.5281/zenodo.215521) with $N=200$ for GPE. See [3,8] for details. For the GPE method, main effect sensitivity indices of an output to each input $x_{i}(i=1, \ldots, 6)$ are calculated as a ratio of variances (Var)

$$
\mathrm{S}_{\mathrm{i}}=\frac{\operatorname{Var}\left[E\left\{f(x) \mid x_{i}\right\}\right]}{\operatorname{Var}\{f(x)\}},
$$

where $E\left\{f(x) \mid x_{i}\right\}$ is the mean effect; that is, the expectation of an output $f(x)$ conditional on an input $x_{i}$, after averaging over the remaining inputs [7,8]. For the PLS method, the relative effect of each of the inputs is calculated using the NIPALS algorithm [9] with $N=100$.

The final method used is the PCE method, where a model output $y=f(\mathbf{x})$ is expanded as a truncated series of orthogonal polynomials, which are functions of the inputs x. After checking, polynomials of order 3 (94 sets of inputs) were deemed to be sufficiently accurate. The global sensitivity index is defined as in equation (2) [10].

\section{Results and Discussion}

\subsection{Effect of varying $g_{e l}$ on the EPD}

Initially, we investigated the effect that changing one of the input conductivities had on the EPD by varying $g_{e l}$ across the range mean $\pm 50 \%$, that is, $1.2 \mathrm{mS} / \mathrm{cm}$ to 3.6

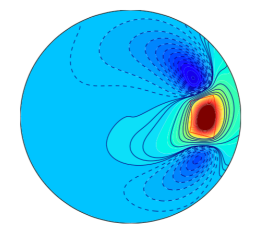

(a) $g_{e l}=1.2 \mathrm{mS} / \mathrm{cm}$ $\min 1 \mathrm{~V}=-0.63, \min 2 \mathrm{~V}=-1.21$ $\max V=4.24$

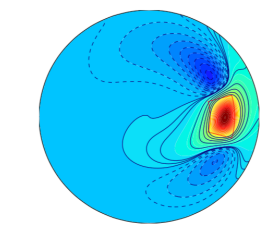

(b) $g_{e l}=2.4 \mathrm{mS} / \mathrm{cm}$ $\min 1 \mathrm{~V}=-0.40, \min 2 \mathrm{~V}=-0.83$ $\max \mathrm{V}=3.20$

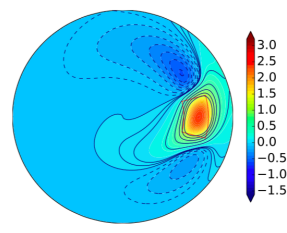

(c) $g_{e l}=3.6 \mathrm{mS} / \mathrm{cm}$ $\min 1 \mathrm{~V}=-0.28, \min 2 \mathrm{~V}=-0.61$ $\max =2.51$
Figure 2. EPDs for $20-70 \%$ ischaemia, with varying values of $g_{e l}$ and mean values for other parameters. Potentials (in $\mathrm{mV}$ ) are given for the main features min1 (lower minimum), min2 (upper minimum) and max (maximum).

$\mathrm{mS} / \mathrm{cm}$. We kept the other parameters at their mean values (Section 2) and considered 50\% ischaemia, from $20 \%$ to $70 \%$. The EPDs are presented as polar plots (Figure 2), where the ventricular surface is 'flattened', with the apex at the centre [3]. In each of cases (a)-(c), the EPD is qualitatively the same; that is, it consists of a maximum, flanked by two minima (and this appears to be the case for all the $50 \%$ ischaemia simulations run). Hereafter, we will refer to the potentials of these features as $\max \mathrm{V}, \min 1 \mathrm{~V}$ and $\min 2 \mathrm{~V}$, respectively, where $\min 1$ is situated below max and $\min 2$ above max (Figure 2).

In Figure 2, as $g_{e l}$ increases, the magnitudes of the outputs $\min 1 \mathrm{~V}(-0.63$ to $-0.28 \mathrm{mV}), \min 2 \mathrm{~V}(-1.21$ to -0.61 $\mathrm{mV}$ ) and $\operatorname{maxV}$ (4.24 to $2.51 \mathrm{mV}$ ) decrease, indicating that changes in $g_{e l}$ have a significant impact on the EPD.

Since previous work, using a subendocardial model of ischaemia [3], has identified ROT as an input to which some outputs are very sensitive, we repeated the above work with ROT varying across the range $60^{\circ}$ to $140^{\circ}$, with the other parameters set to their means. The effect was similar but opposite to that of Figure 2, as $\min 1 \mathrm{~V}, \min 2 \mathrm{~V}$ and max $\mathrm{V}$ all increased in magnitude with increasing ROT.

\subsection{Using GPEs and PLS to determine the effect of varying all six conductivities}

In order to quantify the effect of the other conductivities and also the effect of concurrent changes in more than one conductivity value, we now varied all six conductivities simultaneously, firstly by using GPEs (Section 2). We constructed emulators for three outputs, $\min 1 \mathrm{~V}$, $\min 2 \mathrm{~V}$ and $\max \mathrm{V}$, and from these we produced two types of results. The first is a set of main effect plots (an example for min2V is given in Figure 3), which show the mean effect (Section 2) on the output of varying the six input conductivities. The vertical scale shows the change in the mean value (i.e. 0 represents the mean value, which is $-0.82 \mathrm{mV}$ in this case), across the normalised range of 
Table 1. GPE and PLS sensitivities of three EPD features to the indicated conductivities.

\begin{tabular}{c|c|ccc}
\hline Parameter & Method & min1V & min2V & $\operatorname{maxV}$ \\
\hline$g_{i l}$ & GPE & 0.08 & $\mathbf{0 . 2 9}$ & $\mathbf{0 . 3 1}$ \\
& PLS & -0.26 & $\mathbf{- 0 . 4 9}$ & $\mathbf{0 . 5 5}$ \\
\hline$g_{e l}$ & GPE & $\mathbf{0 . 3 9}$ & $\mathbf{0 . 4 4}$ & $\mathbf{0 . 2 6}$ \\
& PLS & $\mathbf{0 . 6 1}$ & $\mathbf{0 . 6 5}$ & $\mathbf{- 0 . 5 0}$ \\
\hline$g_{i t}$ & GPE & 0.01 & 0.06 & 0.00 \\
& PLS & 0.08 & 0.24 & 0.02 \\
\hline$g_{e t}$ & GPE & 0.09 & 0.00 & 0.12 \\
& PLS & 0.29 & 0.04 & -0.32 \\
\hline$g_{i n}$ & GPE & 0.01 & 0.00 & 0.05 \\
& PLS & 0.09 & 0.02 & -0.22 \\
\hline$g_{\text {en }}$ & GPE & $\mathbf{0 . 4 1}$ & $\mathbf{0 . 2 0}$ & $\mathbf{0 . 2 6}$ \\
& PLS & $\mathbf{- 0 . 6 0}$ & $\mathbf{- 0 . 4 3}$ & $\mathbf{0 . 4 8}$
\end{tabular}

each input. For example, Figure 3 shows min2V varying from approximately $-0.82-0.35=-1.17 \mathrm{mV}$ to $-0.82+0.2=-$ $0.62 \mathrm{mV}$, when $g_{e l}$ varies from 1.2 to $3.6 \mathrm{mV}$, a result that is very similar to the result in Figure 2. Figure 3 also shows that, not only do changes in $g_{e l}$ have a substantial impact on the value of $\min 2 \mathrm{~V}$, but so do changes in $g_{i l}$ and $g_{e n}$, although their effects are less than those of $g_{e l}$ and are in the opposite direction.

One way of summarising these results is via the sensitivity indices that are given in Table 1 for GPE, where significant effects are given in bold. The results in this table quantify the effects shown in Figure 3, with min2V found to be most sensitive to $g_{e l}(0.44)$, then $g_{i l}(0.29)$ and $g_{\text {en }}(0.20)$. PLS sensitivities (Section 2) are also reported in Table 1 and these identify the same inputs to which the outputs are sensitive (although the magnitudes of the two indices are not comparable). The PLS indices have the advantage over the GPE ones in that they are signed, so they indicate the direction of the relationship (as do the main effect plots). Table 1 also shows that $\max V$ is sensitive to the same inputs as $\min 2 \mathrm{~V}$, whereas $\min 1 \mathrm{~V}$ is sensitive to $g_{e l}$ and $g_{e n}$ but not $g_{i l}$. It is also the case that no outputs are sensitive to $g_{i t}, g_{e t}$ or $g_{i n}$. Finally, we note that if the sensitivities for a particular output are totalled, in each case they come to 0.99 , which is very close to 1 and indicates that there are no significant interactions between inputs [8].

Other GP emulators that we constructed were for the position of each of the minima and the maximum (not presented), where we found sensitivity of the feature positions to some input conductivities. We also performed similar simulations, keeping an ischaemic depth of $50 \%$, but starting ischaemia at $10 \%, 30 \%$ and $40 \%$ depth. We found that $\min 1 \mathrm{~V}, \min 2 \mathrm{~V}$ and $\max V$ were generally still sensitive to the same inputs, although, in some cases, the sensitivities were not as strong.

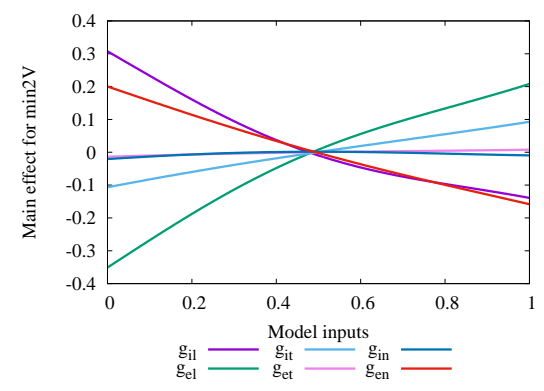

Figure 3. GPE main effect plot for min2V with 20-70\% ischaemia, showing the effect (in $\mathrm{mV}$ for a mean of -0.82) of changes in conductivities.

\subsection{Using PCE to determine the effect of varying all six conductivities}

We now examine the same situation as in Section 3.2 using PCE (Section 2). The mean EPD produced by the emulator is given in Figure 4(a), along with the standard deviation of the potential in (b). Panel (a) is very similar to Figure 2(b) as expected, and panel (b) indicates that the main variation to the conductivities is in $\max \mathrm{V}$, with smaller variations flanking max. Panels (c)-(h) show global sensitivity values (Equation (2)) to the indicated conductivities, across the ventricular surface.

It is immediately clear that there is no EPD sensitivity to $g_{i t}$ and $g_{i n}$, and probably minimal sensitivity of any of $\min 1 \mathrm{~V}, \min 2 \mathrm{~V}$ or $\max V$ to $g_{e t}$ (where we can use Figure 4(a) to locate the features). Next, if we focus on maxV, then Figure 4 suggests that it is sensitive to $g_{i l}, g_{e l}$ and $g_{e n}$, agreeing with the GPE and PLS results (Table 1). However, it is not clear from Figure 4 that sensitivity to $g_{e l}$ is the highest of the three. Then, if we consider the minima, Figure 4 indicates sensitivity of $\min 2 \mathrm{~V}$ to $g_{i l}, g_{e l}$ and $g_{e n}$, matching Table 1, and min1V sensitivity to $g_{e l}$ and $g_{e n}$. However, it is harder to be certain from Figure 4 that min $1 \mathrm{~V}$ is not sensitive to $g_{i l}$, as per Table 1. Another piece of information that does not appear to be something that can be readily determined from Figure 4 is sensitivity to the positions of $\min 1, \min 2$ and max. Finally, we also produced plots of total sensitivity (direct effects of $x_{i}$ as well as interactions of $x_{i}$ with the other inputs [10]), but no significant interactions were found, in agreement with the GPE results. Future work might investigate the utility of the extra information (i.e. not at the maximum and minima) that the PCE approach provides.

\section{Conclusion}

When we compared the methods we were able to draw a number of conclusions. For example, GPE and PLS yield 


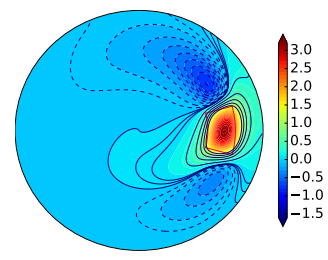

(a) mean

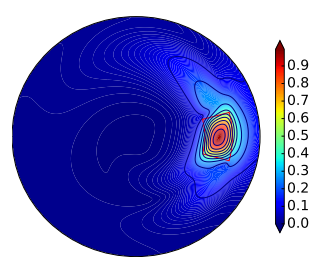

(b) std dev.

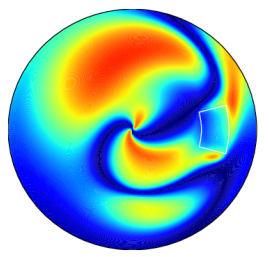

(c) $g_{i}$

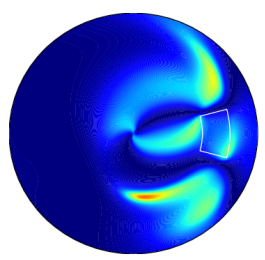

(d) $g_{e l}$

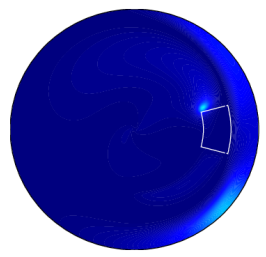

(e) $g_{i t}$

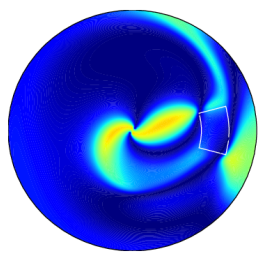

(f) $g_{e t}$

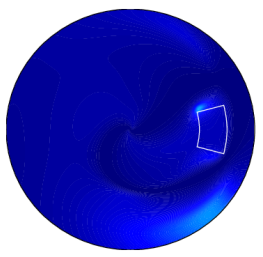

(g) $g_{\text {in }}$

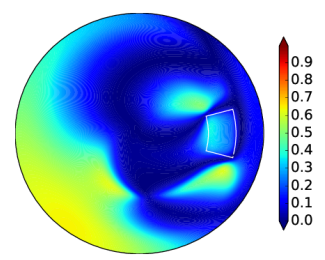

(h) $g_{\text {en }}$

Figure 4. PCE (a) mean EPD and (b) standard deviation of the EPD (mV) for 20-70\% ischaemia. The scale of panel (h) applies to panels (c)-(h) and these panels give global sensitivities to the indicated input conductivities.

the same qualitative results re sensitivity, and, while PCE results are consistent with these, they are harder to interpret. We note that GPE can quantify the effect of input uncertainty unlike PLS, and that PCE provides sensitivities over the whole EPD, whereas GPE and PLS do not. Finally, PCE and PLS have the advantage over GPE that they have lower computational cost. Assuming the results of this work translate to the torso surface in a more complete model, the main conclusion of this work is that, while previous work has indicated $[3,4]$ that the EPD features are most sensitive to ROT and ischaemic depth, there is still significant sensitivity to some of the conductivity values (i.e. $g_{i l}, g_{e l}$ and $g_{e n}$ ). This has implications in terms of personalised medicine because of the differences in the EPDs (and perhaps ECGs), for the same degree of ischaemia, that could result due to variations in conductivity values.

\section{References}

[1] Hopenfeld B, Stinstra JG, MacLeod RS. The effect of conductivity on ST-segment epicardial potentials arising from subendocardial ischemia. Annals of Biomedical Engineering 06 2005;33(6):751-763.

[2] Potse M, Coronel R, Falcao S, LeBlanc AR, Vinet A. The effect of lesion size and tissue remodeling on ST deviation in partial-thickness ischemia;. Heart Rhythm 2007; 4(2):200-206.

[3] Johnston BM, Johnston PR. Determining the most significant input parameters in models of subendocardial ischaemia and their effect on ST segment epicardial potential distributions. Computers in Biology and Medicine 2018; 95:75-89.

[4] Johnston BM, Johnston PR. Sensitivity analysis of ST- segment epicardial potentials arising from changes in ischaemic region conductivities in early and late stage ischaemia. Computers in Biology and Medicine 2018; 102:288-299.

[5] Aras K, Burton B, Swenson D, MacLeod R. Spatial organisation of acute myocardial ischaemia. Journal of Electrocardiology 2016;49:323-336.

[6] Tung L. A Bi-domain model for describing ischaemic myocardial D-C potentials. Ph.D. thesis, Massachusetts Institute of Technology, June 1978.

[7] Johnston BM, Coveney S, Chang ETY, Johnston PR, Clayton RH. Quantifying the effect of uncertainty in input parameters in a simplified bidomain model of partial thickness ischaemia. Medical and Biological Engineering and Computing 2018;56:761-780.

[8] Chang ETY, Strong M, Clayton RH. Bayesian sensitivity analysis of a cardiac cell model using a Gaussian Process emulator. PLoS ONE 2015;10(6):e0130252.

[9] Abdi H. Partial least squares regression (PLS-regression). Encyclopedia for research methods for the social sciences. Sage, 2003; 792-795.

[10] Eck VG, Donders WP, Sturdy J, Feinberg J, Delhaas T, Hellevik LR, Huberts W. A guide to uncertainty quantification and sensitivity analysis for cardiovascular applications. International Journal for Numerical Methods in Biomedical Engineering 2016;32(8):e02755.

Address for correspondence:

Barbara Johnston

School of Environment and Science, and Queensland Micro- and Nanotechnology Centre, Griffith University, Kessels Rd, Nathan, Queensland, Australia, Barbara.Johnston@griffith.edu.au 